\title{
In-Band Pumped Conical Refraction Nd:KGW Laser
}

\author{
C. Howlader ${ }^{1}$, T. Waritanant ${ }^{1}$, K.A. Fedorova ${ }^{2}$, G.S. Sokolovskii ${ }^{3}$, E.U. Rafailov ${ }^{2}$, A. Major ${ }^{1}$ \\ ${ }^{1}$ Department of Electrical and Computer Engineering, University of Manitoba, Winnipeg, R3T 5V6, Canada \\ ${ }^{2}$ School of Engineering \& Applied Science, Aston University, Birmingham, UK \\ ${ }^{3}$ Ioffe Institute, St. Petersburg, Russia
}

\begin{abstract}
We have demonstrated an in-band pumped conical refraction (CR) Nd: KGW laser. The CR laser was diode-pumped at $910 \mathrm{~nm}$ and produced an output power of $1.15 \mathrm{~W}$ at $1069 \mathrm{~nm}$.
\end{abstract}

Keywords - conical refraction; diode-pumped; Nd-lasers.

\section{INTRODUCTION}

The conical refraction phenomenon was predicted by Hamilton and after that experimentally demonstrated by Lloyd [1]. Conical refraction happens once light is propagating along one of the optical axes of a biaxial crystal. Conicity of the emerging light depends on the length of the crystal and its refractive indices. Another condition to clearly observe $\mathrm{CR}$ is that the ratio of the $\mathrm{CR}$ ring radius to the incident beam waist radius should be much greater than one [1]. CR has the potential application in the fields of optical trapping, free space communication, super-resolution microscopy, quantum computing, cryptography, polarization demultiplexing and multiplexing, polarimetry and mode conversion [2-4]. The output power of the CR Nd: KGW lasers, however, is limited by moderate thermal conductivity of the crystal. This issue can be overcome by in-band pumping which significantly reduces heating due to quantum defect [5-8]. In this work we have demonstrated the first CR Nd:KGW laser with in-band pumping at $910 \mathrm{~nm}$ instead of traditional $808 \mathrm{~nm}$ [9]. In the initial experiments the laser operated at $1069 \mathrm{~nm}$ and produced an output power of $1.15 \mathrm{~W}$.

\section{EXPERIMENTAL SETUP AND RESULTS}

Fig. 1(a) shows the schematic diagram of a laser cavity containing the Nd: KGW gain medium which was cut along one of the optical axes to get CR [9]. The crystal was $2 \times 4.2 \times 18 \mathrm{~mm}-$ long, had $3 a t \%$ Nd-ion doping, and was antireflection coated at the laser and pump wavelengths. The crystal was wrapped in indium foil and held by a water-cooled $\left(16{ }^{\circ} \mathrm{C}\right)$ metallic holder. The pump at $910 \mathrm{~nm}$ was provided by a fiber coupled laser diode which had $105 \mu \mathrm{m}$ fiber core diameter and numerical aperture of 0.22. A dichroic mirror (DM) was used to introduce the pump light into the cavity which was designed taking into account thermal lensing [10]. L1, L2 and L3 distances were 24 $\mathrm{mm}, 398 \mathrm{~mm}$ and $439 \mathrm{~mm}$, respectively, and produced a beam diameter of $550 \mu \mathrm{m}$ in the crystal. M1 is a concave mirror $(\mathrm{r}=500 \mathrm{~mm})$ and the output coupler (OC) had 2.4\% transmission. A red He-Ne laser was used to observe a conical refraction ring pattern at the plane of the DM using a CCD camera. The propagation direction of the $\mathrm{He}-\mathrm{Ne}$ laser was aligned to be collinear with the generated laser light, thus ensuring alignment close to the $\mathrm{CR}$ condition. A further careful adjustment of cavity mirrors could be used to operate the laser in the CR mode as well as in the normal non-CR regime. A transition between two regimes was accompanied by the change in the position of the generated laser mode at the DM. In addition, in the non-CR regime lasing took place at $1067 \mathrm{~nm}$ and in the CR regime - at $1069 \mathrm{~nm}$. In both cases laser had an excellent beam quality and produced maximum output powers of $1.8 \mathrm{~W}$ and $1.15 \mathrm{~W}$, respectively. The threshold pump powers were measured to be $0.55 \mathrm{~W}$ for $1067 \mathrm{~nm}$ and $1.52 \mathrm{~W}$ for 1069 $\mathrm{nm}$ lasing. Fig 1(b) shows the output power versus the absorbed power for both regimes with the slope efficiencies of $46 \%$ and $32 \%$ for $1067 \mathrm{~nm}$ and $1069 \mathrm{~nm}$ lasers, respectively. Lower output power in the CR regime can be explained by the laser beam walk-off and agrees with the previous report [11]. The output beam did not produce the CR ring pattern in the current setup due to a big mode size in the crystal. This will be addressed in the future experiments.

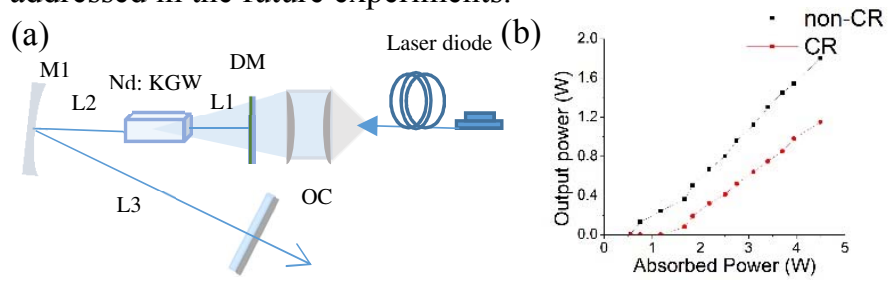

Fig. 1: (a) Experimental setup of the Nd:KGW CR laser and (b) its output power vs the absorbed pump power.

In summary, a simple three mirror cavity CR laser with inband pumping was demonstrated. The laser was also operated in the standard regime which had a higher slope efficiency than the CR laser. Further experiments will include optimization of the pump spot to produce a clear CR pattern and possibly nonlinear frequency conversion [12-15].

\section{REFERENCES}

[1] M.V. Berry, M.R. Jeffrey and J.G. Lunney, Proc. Soc. A 462, 1629 (2006).

[2] A. Brenier, Appl. Phys. B 122, 277 (2016).

[3] A. Turpin, Yu. V. Loiko, T. K. Kalkandkiev, H. Tomizawa, and J. Mompart, Opt. Lett. 39, 4349 (2014).

[4] G. S. Sokolovskii, V. Yu. Mylnikov, S. N. Losev, K. A. Fedorova, E. U. Rafailov, Proc. SPIE 10090, 100901R (2017).

[5] R.C. Talukder, M.Z.E. Halim, T. Waritanant, and A. Major, Opt. Lett. 41, 3810 (2016).

[6] T. Waritanant and A. Major, Appl. Phys. B 122, 135 (2016).

[7] T. Waritanant and A. Major, Opt. Express 24, 12851 (2016).

[8] M.Z.E. Halim, R.C. Talukder, T. Waritanant, and A. Major, Laser Phys. Lett. 13, 105003 (2016).

[9] A. Abdolvand, K.G. Wilcox, T.K. Kalkandjiev, and E.U. Rafailov, Opt. Express 18, 2753 (2010)

[10] H. Mirzaeian, S. Manjooran, A. Major, Proc. SPIE 9288, 928802 (2014).

[11] R. Cattoor, I. Manek-Honninger, D. Rytz, L. Canioni, M. Eichhorn, Opt. Lett. 39, 6407 (2014).

[12] R. Akbari and A. Major, Laser Phys. 23, 35401 (2013).

[13] H. Zhao, I. T. Lima, and A. Major, Laser Phys. 20, 1404 (2010).

[14] I.T. Lima Jr., V. Kultavewuti, and A. Major, Laser Phys. 20, 270 (2010).

[15] S. Manjooran, H. Zhao, I. T. Lima, A. Major, Laser Phys. 22, 1325 (2012). 